\title{
Design and Implementation of Campus Competition Information Sharing Platform Based on Android
}

\author{
Xiaobo Ming ${ }^{1}$, Haizhen $\mathrm{Pan}^{2}$, Yun $\mathrm{Wu}^{3}$ \\ ${ }^{1}$ Shangrao Vocational Technical College, Jiangxi, Shangrao, 334001; \\ ${ }^{2}$ Shangrao Normal University, College of Mathematics and Computer Science, Jiangxi, Shangrao, 334001; \\ ${ }^{3}$ Guizhou University, College of Computer Science and Technology, Guizhou, Guiyang, 550025
}

\begin{abstract}
At present, the market share of the Android system in the smartphone operating system has reached $90 \%$. In view of the limitations of the existing sharing platform for campus competition information, a campus APP, which integrates competition and activity information release, information sharing and interrogative interaction, is put forward. In this paper, the overall architecture design, function module design, database design and RESTful API design are carried out. The Java language and MVP pattern are used by Android part. Python language, RESTful architecture and ORM technology are used at the backend to realize the information of publishing competition information, the list of competition information, the information of search competition and the management of competition information. The platform is running on the North Campus of Guizhou University, the current operation situation is well.
\end{abstract}

Keywords: Campus competition information; Android; MVP pattern; RESTful architecture; ORM technology.

\section{Introduction}

At present, enterprises and institutions in our country have higher and higher requirements for the comprehensive practical ability of college students. Participating in professional competitions and activities during the university period is an important way to improve students' comprehensive practical ability and enrich campus life[1]. The platforms for publishing and sharing campus competition information and activity information include QQ group, super curriculum schedule and saikr, etc. The QQ group has simple functions, limited information and messy information. The super curriculum schedule takes the curriculum schedule as the main body and involves all kinds of information on campus and lacks professionalism. Saikr is facing the national competition platform for college students, which has space limitations.

According to the market share data of smart phone

Received date: 2018-00-00

Foundation program: Theory and Practice of Integrating Network Resources to Improve the Teaching Effect of Computer Culture Foundation (Provincial Education Reform Project, JXJG-11-16-5); College Entrance Examination" filling applications" Platform (The 2017 Municipal Science and Technology Plan Project, 17A014); Research on Training Mode of Undergraduate Tutorial Talents' Innovative Ability (Gdgj2016008)

Author's introduction: Pan Haizhen (1978-), female, lecturer, The research direction is computer information technology, e-commerce teaching and industry-university-research cooperation; Ming Xiaobo (1975-), male, associate professor, The research direction is computer network application and e - commerce; +Corresponding Author: $\mathrm{Wu}$ Yun(1973-), male, doctor, associate professor, master tutor, Institute Director of Computer Simulation in Guizhou University, The research direction is recommendation system, distributed computing and application of big data technology.

(C) The Authors, published by EDP Sciences. This is an open access article distributed under the terms of the Creative Commons Attribution License 4.0 
patterns, they have similar ideas. Controller / Presenter is responsible for logical processing, Model is responsible for providing data, and View is responsible for displaying. In Android projects, Activity and Fragment occupy most of the development work. MVP pattern is the code used to optimize activity and fragment. The advantage of MVP pattern is that the model is completely separated from the view, it is not affected by the model when modifying the view, and the model can be used more efficiently[4] .

(2) RESTful architecture

REST[5,6] (Representational State Transfer, which represents the layer state transition) is a set of architectural constraints and principles. An application or design that meets these constraints and principles is RESTful, and an API that conforms to REST architecture is called RESTful API. The RESTful API has three important features [7,8]: First, make full use of the semantics of the HTTP protocol itself to regulate the use of request actions. RESTful uses the standard HTTP method and there are four commonly used methods: GET, POST, PUT and DELETE. Second, exposed resources exist, and the URI transparently displays the requested resource. Third, the RESTful API is stateless, regardless of context and current state, greatly reducing complexity.

(3) ORM technology

ORM (Object Relational Mapping) is used to implement the conversion between data of different types of systems in the object-oriented programming language [9]. In effect, ORM technology can create virtual object database in programming language and add, delete, modify and query mapping objects. There is no need to write SQL statements, which greatly simplifies program development and effectively avoids SQL injection vulnerabilities.

\section{Design of campus competition information sharing platform}

\subsection{Overall architecture design}

This platform is developed with a layered architecture, which can reduce the coupling degree between layers as much as possible to ensure that there will be no mutual interference in the development process and improve work efficiency. The overall architecture design of this platform is shown in figure 1 . It can be seen from the figure that the system architecture is divided into a Data Layer and an APP Layer. The back-end database uses MySQL, the development language uses Python, the database operation uses Python's third-party library SQLAlchemy, the back-end service uses Python's Web application framework Flask, and the unit test uses Python's third-party library Nose. Android is developed in Java language and adopts SSM framework and MVP design mode. The RESTful API is used for data interaction between Android and back-end.

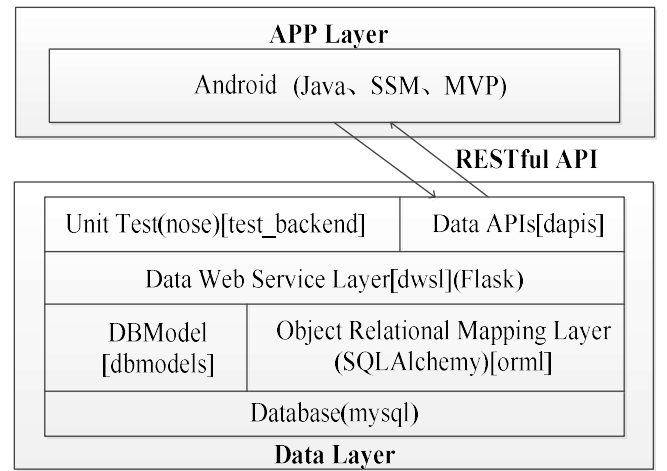

Figure 1. Overall architecture design diagram.

\subsection{Functional module design}

The campus competition information sharing platform based on Android includes a client module and a background management module, and its functional module design is shown in figure 2.

(1) Client module. The main functions of the client module include user registration, user login, password retrieval, classified display of competition information, search for competition information, application for release authority, release of competition information, my attention, my collection, my information, information settings, viewing of competition details, etc.

(2) Background management module. The main functions of the background management module include user login, user management, system parameter management, competition information management, statistical analysis, etc.

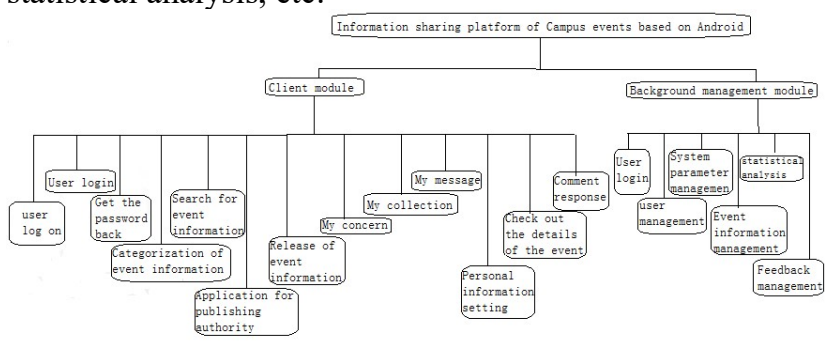

Figure 2. Function module design diagram.

\subsection{Database design}

According to the functional module design of the campus competition app, it is determined that the main entities in the platform are users, user information, search records, application information, collection information, forwarding information, praise information, comment information, competition information and competition tags. Part of the entity-relationship of the platform is shown in figure 3 . 


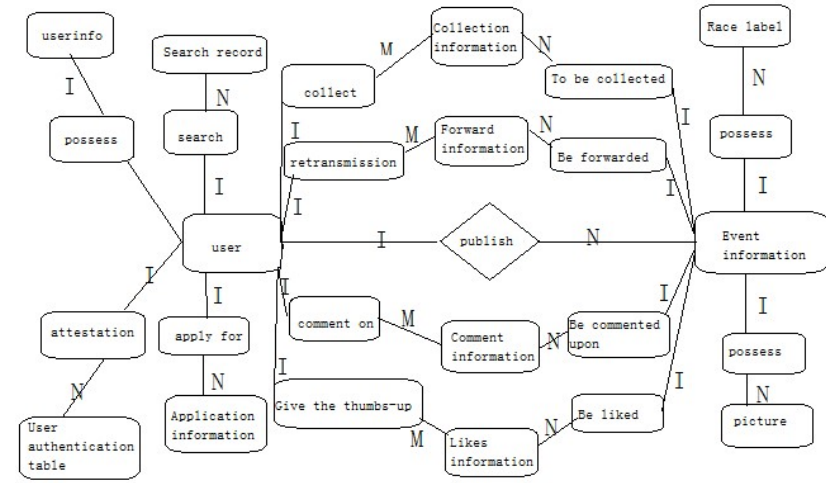

Figure 3. Platform entity - contact diagram.

According to entity-attribute analysis, the database table of the platform mainly includes user table, user information table, user authentication table, search record table, application information table, competition information table, competition label table, classification table, picture table, attachment information table, attention information table, collection information table, forwarding information table, praise information table and comment table. The fields in the competition information table include competition number, competition theme, competition release unit, registration or competition time, competition description, competition link, release time, classification number and user number. Competition number is primary key, category number and user number are foreign keys.

\subsection{API design}

This platform uses RESTful architecture style to design API. RESTful abstracts everything on the network into resources, each resource has a unique resource identifier URI. There are 46 APIs designed for the campus competition APP, including displaying the competition information list on the home page, applying for secondary administrator, forwarding the competition information, commenting on the competition information, displaying the competition information details, keyword search, tag search, publishing the competition information, displaying my favorite competition, super administrator reviewing the competition information and so on.

Due to limited space, we will only introduce the API design for displaying the competition information list on the home page, as shown in figure 4.

\begin{tabular}{|c|c|}
\hline Address & /v 1/matchinfo/all/show/ \\
\hline $\begin{array}{l}\text { Request } \\
\text { mode }\end{array}$ & GET \\
\hline $\begin{array}{l}\text { Required } \\
\text { parameter }\end{array}$ & $\begin{array}{l}\text { \{"usertoken_str":token, "matchinfo_label": "Competition", } \\
\text { "matchinfo_startposi"::0, "matchinfo_pagesize": 10\} }\end{array}$ \\
\hline $\begin{array}{l}\text { API retum } \\
\text { value } \\
\text { enumeration }\end{array}$ & $\begin{array}{l}\{\text { "infostatus":True, "infomsg": }\{\text { "page_number": } 3\} \text {, "inforesult": } \\
\{\text { "matchinfo_id": } 1 \text {, "uesr_head": "head.jpg", "user_name": "Zhang } \\
\text { San", "follow_info": "Attention TA", "matchinfo_favorite": } 0 \text {, } \\
\text { "matchinfo_praise":0, "favorite_number": } 20 \text {, "praise_number": } 30 \text {, } \\
\text { "forward_number": } 40 \text {, "comment_number": } 50 \text {, "matchinfo_title": } \\
\text { "Computer Program competition", "matchinfo_unit": "Computer } \\
\text { college", "matchinfo_desp": "2017/04/20 22:00", "matchinfo_desp": } \\
\text { "The computer program competition mainly trains students' } \\
\text { programming ability", "match_publishtime": "Five minutes ago"\}]\} }\end{array}$ \\
\hline
\end{tabular}

Figure 4. API design for displaying competition information list.

\section{Implementation of campus competition information sharing platform}

According to APP requirements, overall architecture design, database design and RESTful API design, Python language, ORM and Flask framework are used in the back end, and Java language, SSM framework and MVP pattern are used in the mobile end to encode and implement all functions in the platform function module design. Due to space constraints, the following only describes the Android end and back end implementation processes for displaying the competition list.

\subsection{Implementation of Android side for displaying competition list}

The interface for displaying the competition information list is shown in figure 5.

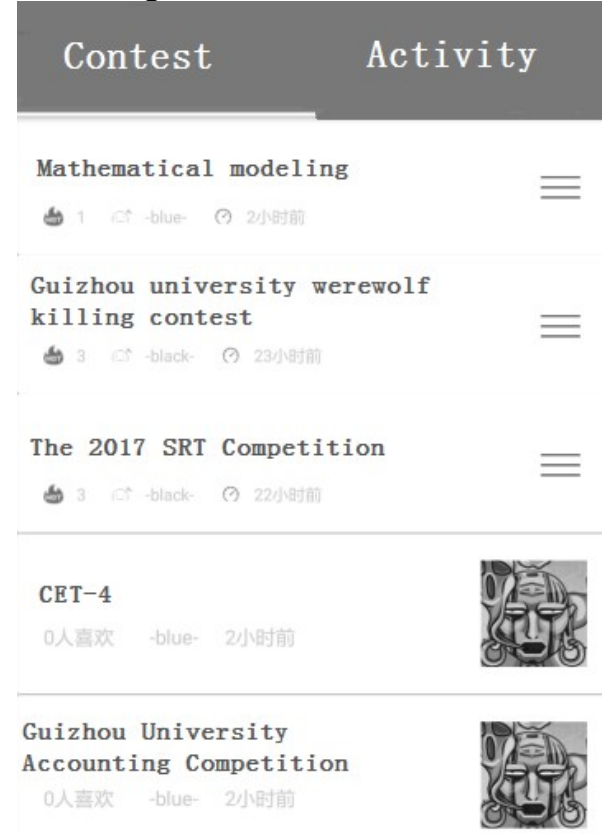

Figure 5. Interface for displaying competition information list. 
The Android code implementation process for displaying the competition information list is shown in figure 6 .

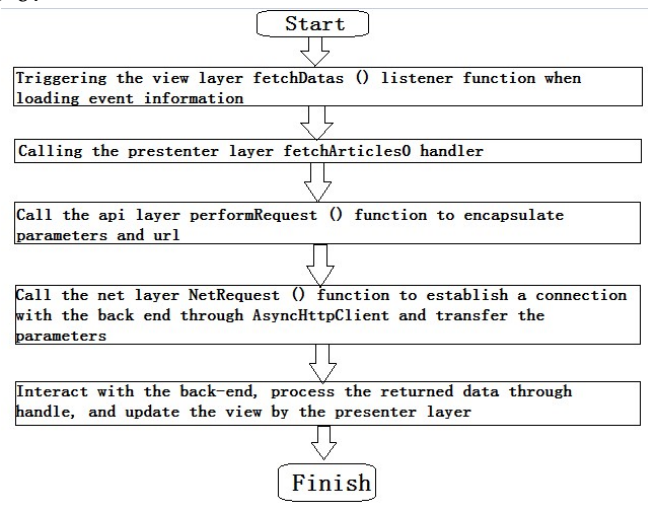

Figure 6. Android side code implementation flow chart.

In MVP pattern, Presenter completely separates Model from View, and the main program logic is implemented in Presenter. Part of the code that implements the Presenter layer function fetchArticles ( ) displayed in the competition information list is as follows:

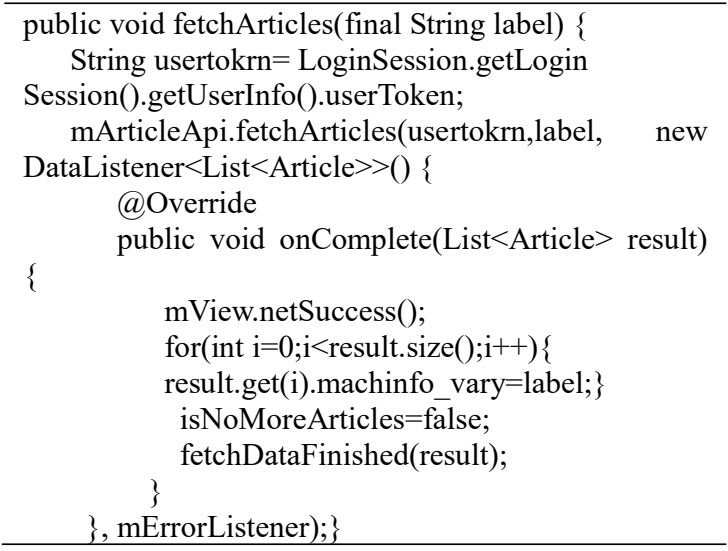

\subsection{Implementation of back-end for displaying competition list}

The back-end code implementation process for displaying the competition information list is shown in figure 7.

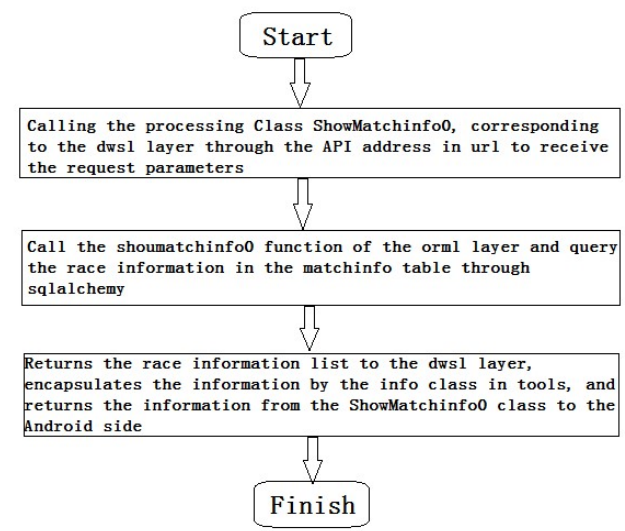

Figure 7. Back - end code implementation flow chart.

The back-end orml layer implements ORM technology through Python's third-party library SQLAlchemy. The match info table needs to be queried to display the competition information, which can be realized by using the query ( ) function of SQLchemy session. Part of the code for the show match info ( ) function is as follows:

matchinfolist $=$ session.query(Matchinfo.match info id,Matchinfo.matchinfo title,Matchinfo.matchinf o_unit,Matchinfo.matchinfo_time,Matchinfo.matchinf o desp,Matchinfo.matchinfo publishtime,Matchinfo. matchinfo_userid).filter_by(matchlabel_labelid=matc hinfolabel).all()

\section{Conclusion}

According to the actual needs of college students, this paper designs the overall architecture, functional modules, database and RESTful API of the campus competition information sharing platform. Android uses Java language and MVP pattern, while Python language, RESTful architecture and ORM technology are used in the back end to realize the functions of publishing event information, displaying event information list, searching event information and event information management. The platform has been put into trial operation in the north campus of Guizhou University and is now in good condition.

\section{References}

1. Yu Zhifeng. Design and Implementation of College Students' Computer Competition Platform [D]. Northeastern University, 2015.

2. Gartner. Gartner Says Worldwide Sales of Smartphones Recorded First Ever Decline During the Fourth Quarter of 2017 [EB/OL].

https://www.gartner.com/newsroom/id/3859963, 2018-02-22/ 2018-05-10.

3. Hao Jie. Design of Information Platform for College Student Associations Based on MVP Model [J]. Journal of Harbin Institute of Vocational Technology, 2017(06):105-107.

4. Guo Jianing. Design and Implementation of Front End Framework CAS Front Based on MVP [D]. Tianjin University, 2016.

5. FIELDING R T. Architectural styles and the design of network-based software architectures [D]. University of California, Irvine, 2000.

6. Qin Guo, He Tao. Research on REST Application in Heterogeneous Mobile Client Access [J]. Computer Applications and Software, 2015, 32(4):77-79.

7. Zhang Wumei. Design and Implementation of Resource Management System Based on RESTful Web Technology $[\mathrm{J}]$. Computer Applications and Software, 2014, 31(05):24-28

8. Wu Yanbiao, Xiong Yong, Yao Wei, etc. Application of Smart Home System Based on RESTful Web [J]. Journal of computer application, 2015, 35(S2):284-289.

9. Yan Chengwu. Design and Implementation of ORM Framework Supporting the Separation of Library and Table and Reading and Writing [D]. Harbin Institute of Technology, 2016. 